\title{
Doping-Dedoping-Driven Optic Effect of $\pi$-Conjugated Polymers Prepared in Cholesteric-Liquid-Crystal Electrolytes
}

\author{
Hiromasa Goto \\ Graduate School of Pure and Applied Sciences, Institute of Materials Science, University of Tsukuba, \\ Tsukuba, Ibaraki 305-8573, Japan \\ (Received 25 September 2006; revised manuscript received 21 March 2007; published 22 June 2007)
}

\begin{abstract}
Chiral conjugated polymers were synthesized by electrochemical polymerization in a cholestericliquid-crystal (CLC) electrolyte. The polymers display a characteristic finger print texture similar to that of CLC. The natural optical activity of the polymers was successfully controlled by the electrochemical doping-dedoping procedure. The finding of this unprecedented mechanism, involving doping of $\pi$-conjugated systems, can be an important advance in the development of plastic optoelectronics based on conductive polymers.
\end{abstract}

DOI: 10.1103/PhysRevLett.98.253901

PACS numbers: 42.70.Jk, 81.05.Lg, 82.35.Cd, 82.45.Aa

Conductive polymers have promising utility as the basis for plastic electronics and have been developed practically as solar cells, high-performance capacitors, fieldeffect transistors, organic electroluminescence devices, and electrochromic devices [1]. In the neutral state, conjugated polymers are inherently insulators. However, doping by addition of small amounts of electron donors or acceptors has been shown to provide an impressive increase in conductivity. In the case of polyacetylene films prepared by the Shirakawa method, a conductivity increase of more than $10^{7}$ times can be achieved by vapor doping with iodine [2]. Conductive polymers also undergo a color change accompanying the electrochemical oxidation and reduction by doping or dedoping of a counterion. This electrochemically induced transition is utilized in electrochromic devices, where alternating voltages are applied progressively across a conductive polymer film [3].

The preparation of conductive polymers can be achieved by chemical polymerization using a transition-metal catalyst, or by electrochemical polymerization. In the latter process, the polymer film is grown epitaxially on an electrode surface, typically in an isotropic liquid such as acetonitrile or tetrahydrofuran [4,5]. Polymer films produced by electrochemical polymerization can be electroactive, yet do not usually exhibit either linear or circular dichroism. However, it is shown in the present study that it is possible to induce the formation of chiral structure in the growing polymers by performing the process in a cholesteric-liquid-crystal (CLC) electrolyte.

CLC has structural chirality and thus exhibits strong optical rotary power. The helical arrangement between pseudolayers of CLC on a macromolecular scale greatly enhances the physical manifestation of chiroptical property, to such an extent that the optical rotation of a chiral molecule can be enhanced by up to $10^{4}$ times in the CLC phase $[6,7]$. These optical properties have recently been confirmed for cholesteric gels [8]. Therefore, it is expected that chiral conductive polymers with CLC like macromolecular formation show quite large optical activity in the range of ultraviolet-visible-near infrared.

Early observations of the optical activity of copper coil helices in the microwave region revealed that the optical activity absorption bands are simply related to property of the wire in the helix [9], demonstrating that helical formations are capable of exhibiting optical activity dependent on the helical spacing or periodicity. CLC functions in a manner analogous to a microcopper coil, where the chiral order parameter can be tuned by changing the helical pitch. The helicity of a chiral polymer in molecular level can be controlled by an external force such as an electric or magnetic field to realized tunable optical activity. In the present study, such tunable optical activity is demonstrated for a chiral polymer prepared from an achiral monomer by electrochemical polymerization using CLC as a chiral electrolyte.

The optical texture and schema of constituents of the CLC electrolyte system in this study are shown in Fig. 1. The CLC electrolyte is prepared by addition of a CLC inducer with axial chirality $\left[1,1^{\prime}\right.$-binaphthyl-2,p-(trans-4-pentylcyclohexyl)phenoxy-1-hexyl] ether (PCH506-binol) $(0.02$ g) [10,11] to 4-cyano-4'-hexyl biphenyl (6CB) (0.5 g), a well-known nematic LC [12]. Liquid crystallinity is confirmed to be maintained after addition of tetrabutylammonium perchlorate (TBAP) $(5 \mathrm{mg})$ as a supporting salt and monomer [2,5-bis- $\left(2,2^{\prime}\right)$ bithiophenyl5 -yl-benzoic acid dodecyl ester] $(2 \mathrm{mg})$. The mixed system exhibits a fan-shaped texture at room temperature, typical of the cholesteric phase. 6CB is thermotropic liquid crystals and can be regarded as a solvent with fluidity, while the addition of a supporting salt provides ionic conductivity. The mixture of LC, monomer, CLC inducer, and supporting salt can be used as an electrolyte for electrochemical polymerization in place of normal systems containing a supporting salt in isotropic solution. 

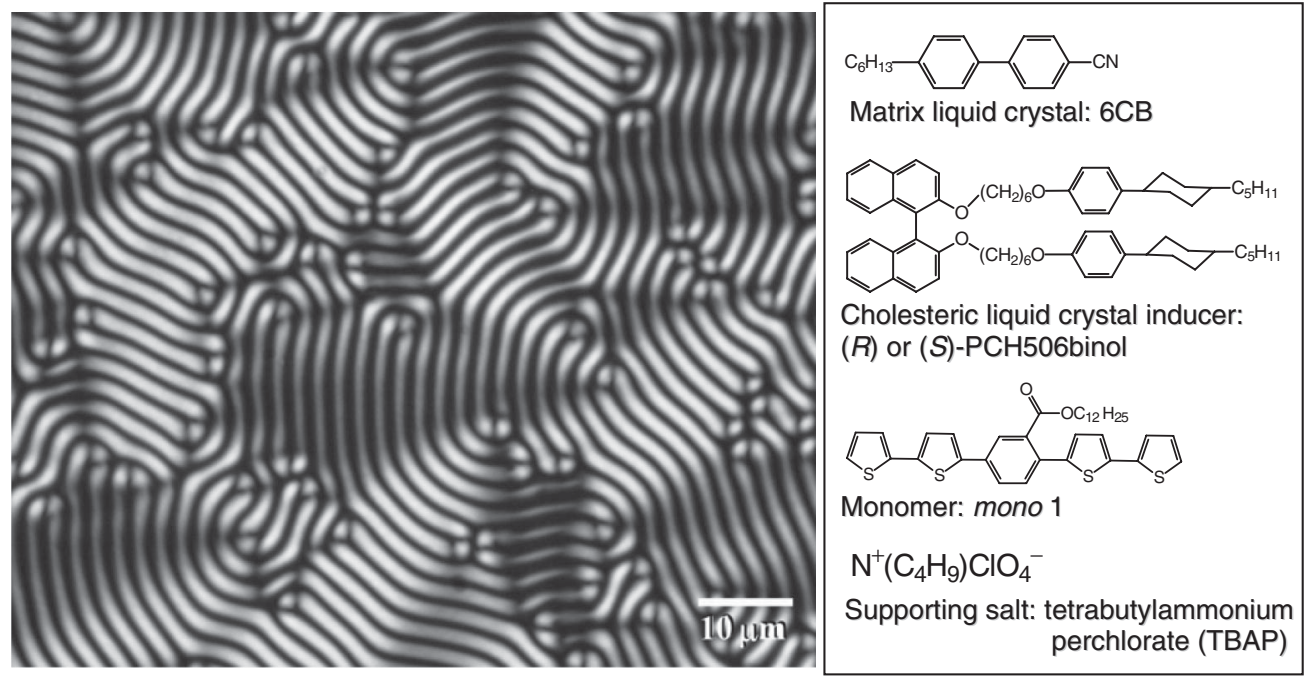

FIG. 1. Polarizing optical microscopy image of the cholesteric-liquid-crystal (CLC) electrolyte at $15^{\circ} \mathrm{C}$ (left) and schema of constituents (right).

The electrolyte solution in a vial was heated once to $40{ }^{\circ} \mathrm{C}$ to completely dissolve the supporting salt, monomer, and CLC inducer in the 6CB as the LC solvent. Visual inspection with polarizing optical microscopy (POM) confirms that the CLC electrolyte containing the monomer exhibits uniform thermotropic cholesteric liquid crystallinity at room temperature. The distance between stripes in the texture corresponds to the helical half-pitch of cholesterics. Electrochemical polymerization was carried out using this CLC electrolyte containing monomer. The CLC mixture was injected between sandwiched indium oxide (ITO) coated glass electrodes using a Teflon sheet (thickness: $0.19 \mathrm{~mm}$ ) as a spacer. The reaction cell was heated to $40^{\circ} \mathrm{C}$ and then gradually cooled to $15^{\circ} \mathrm{C}$ to obtain a good fingerprint texture. A voltage of $4 \mathrm{~V}$ was then applied to the cell. The optical texture of the CLC mixture remained unchanged upon voltage application. The polymerization temperature was maintained at a constant $15{ }^{\circ} \mathrm{C}$ through the use of a custom-made temperature control stage with Peltier element in order to preserve the CLC phase. After $30 \mathrm{~min}$, an insoluble and infusible polymer thin film of $210 \mathrm{~nm}$ in thickness coated the anode side of the ITO electrode [13]. After washing with methanol, water, acetonitrile, methanol, water, and acetone in order, the polymer film on the ITO was dried under reduced pressure. Two polymers, abbreviated poly1 and poly2, were prepared in the CLC electrolyte containing the CLC inducer PCH506-binol of the $(R)$ or $(S)$ configuration, respectively.

The polymers thus prepared exhibit characteristic fingerprint texture very similar to that of cholesterics under POM observations with transmission light, as shown in Fig. 2. However, the film surface displays no characteristic structure under POM observations with reflection light. This is due to the fact that the individual macromolecular main chains are arranged in a helix like the CLC electrolyte. In this polymerization process, three-dimensional molecular imprinting in the helical continuum of the CLC electrolyte would be performed. Although the molecular structure of the polymers is completely different from that of the CLC electrolyte, the form of helical molecular arrangement is quite similar to that of the CLC electrolyte. The individual main chains of the polymer are arranged by the topological imprinting of structural chirality in the course of the epi-

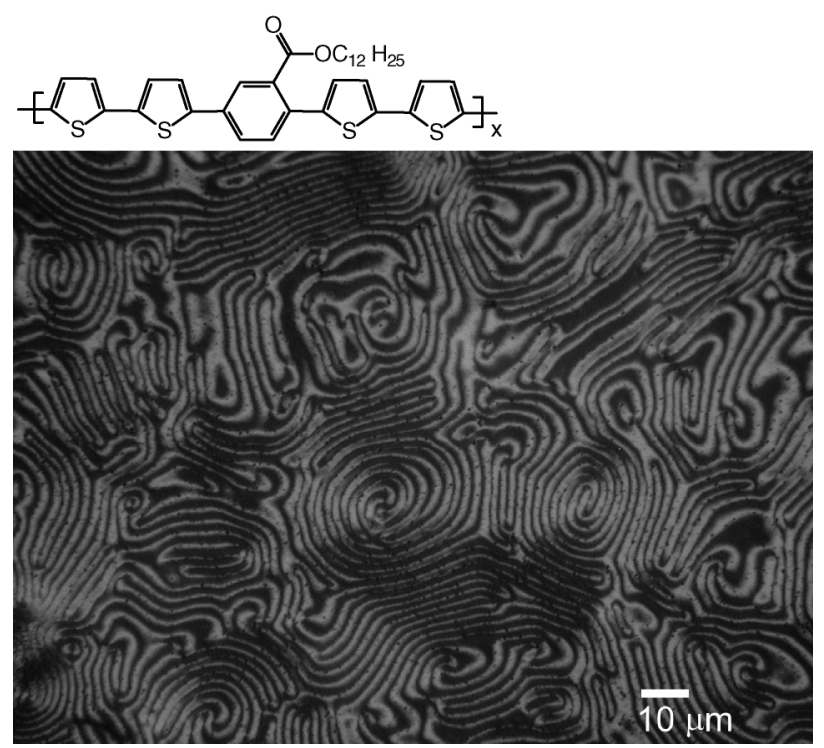

FIG. 2. Molecular structure (top) of poly1 and poly2; both polymers have the same molecular structure but opposite chirality, and optical texture of the cholesteric electrolyte free poly 1 under a polarizing optical microscopy observation with transmission light (lower). Poly2 shows the same fingerprint texture as poly1. Poly1 was prepared in the CLC electrolyte containing $(R)$-PCH506binol. Poly2 was prepared in the CLC electrolyte containing $(S)$-PCH506binol. 
taxial electrochemical polymerization in the one-handed helically twisted matrix of the CLC electrolyte.

Absorption and circular dichroism (CD) spectra for the polymer films were obtained under application of voltages with the electrochemical method in $0.1 M$ TBAPacetonitrile solution (in the absence of monomer, chiral inducer, and liquid crystals). The cell included a platinum wire as the counter electrode, an $\mathrm{Ag} / \mathrm{Ag}^{+}$reference electrode (the reference electrode was calibrated by $\mathrm{Fc} / \mathrm{Fc}^{+}$), and the polymer deposited on the ITO. The absorption spectrum of oxidized poly1 and poly2 exhibits a peak at $440 \mathrm{~nm}$ accompanied by a weakening of the $\pi-\pi^{*}$ transition of the main chain and a strengthening of the absorption bands at $700 \mathrm{~nm}$ (Fig. 3, bottom) and 1200-1300 nm due to generation of radical cations and dications on the main chain. The color of the polymer also changed from orange to dark blue upon oxidation.

This is typical behavior for electroactive conducting polymers [1,14]. As shown in Fig. 3 (top and middle), both poly1 and poly 2 exhibited complementary strong mirror-image Cotton effect in the $\pi-\pi^{*}$ transition region of the polymer main chain in the reduced state, indicating that the two polymers have the same degree of chirality but

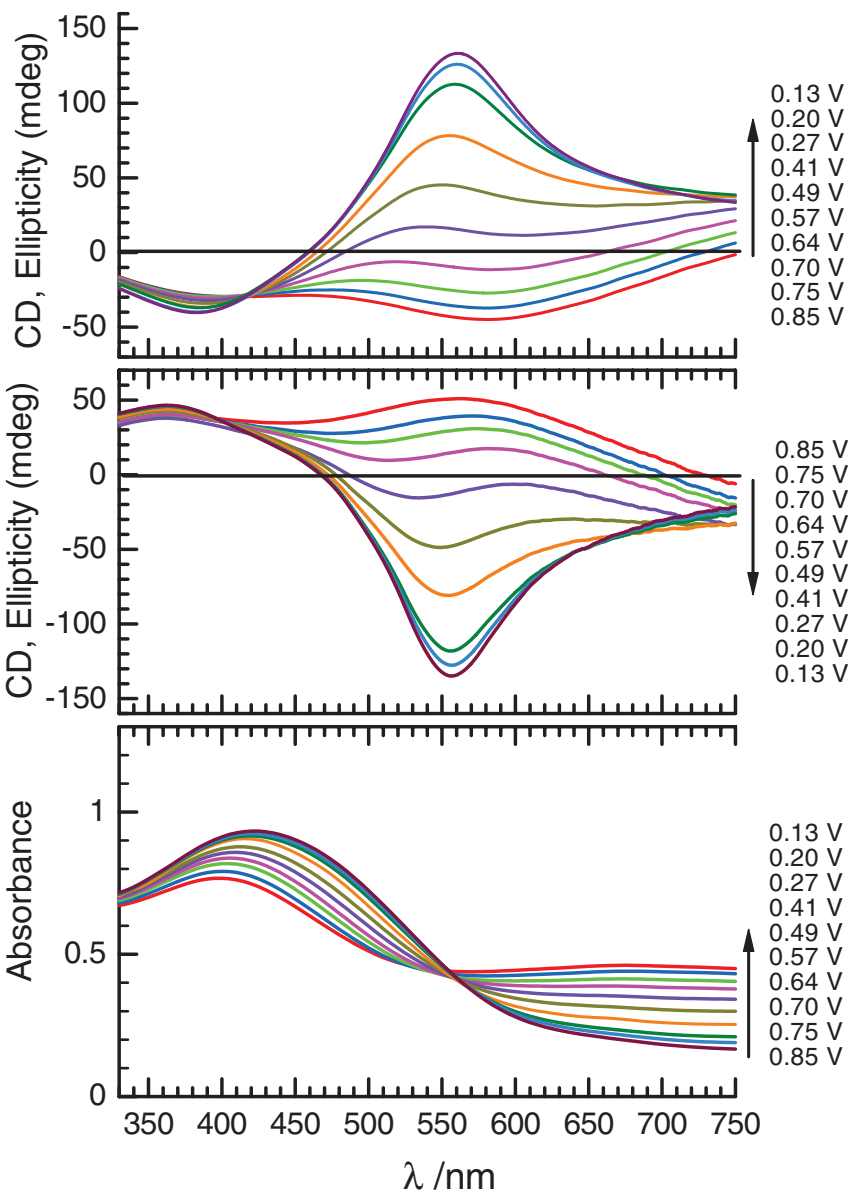

FIG. 3 (color). CD spectra of poly1 (top) and poly2 (middle), and absorption spectra of poly1 (bottom) as a function of applied voltages $\mathrm{vs} \mathrm{Fc} / \mathrm{Fc}^{+}$in $0.1 M \mathrm{TBAP} /$ acetonitrile solution. opposite sense. The zero crossing point of the $\mathrm{CD}$ spectra is located near the position of the peak in the absorption spectra, suggesting that the electrochemical polymerized polymer forms a chiral aggregate. Such chiral aggregation has been reported previously for a polymer having chiral substituents, the polymer prepared from chiral monomer, in an isotropic solvent [15]. The resulting polymers in this study are considered consistent with the reported model, however the $\mathrm{CD}$ intensity is quite larger. This is due to the fact that poly 1 and poly 2 form a CLC-like helical macrostructure.

In the oxidized state, the CD spectra of the polymers displayed a decrease in intensity and an inversion in the sign of the $\mathrm{CD}$. The complementary mirror-image relationship between poly 1 and poly 2 cannot be due to the CLC inducer employed in polymerization because the Cotton effect of the CLC inducer, PCH506binol, is only observed at wavelengths in the range $240-340 \mathrm{~nm}$. The reversible inversion of the sign of the Cotton effect at $560 \mathrm{~nm}$ in the doping-dedoping process via change in the electronic state of the polymer indicates that the polymers have an inherently chiral structure.

Application of $0.13 \mathrm{~V}$ for dedoping (reduction) of perchlorate ion out of the polymer allowed the restoration of these peaks to the original intensities observed in both the absorption and CD spectra. This result indicates that the optical properties of the polymers can be changed by adjusting the conditions of electrochemical dopingdedoping process. The electrochemical doping-dedoping process did not induce any distinct change in the optical texture or surface structure of the polymer but the color of the optical texture was reversibly changed.

The CD spectra for polymers having chiral substituent in poor solvents and in the form of cast film have been reported as reflecting the effects of exciton coupling, usually involving Davydov splitting [16-23]. Exciton coupling requires the presence of an unconjugated chromophore in the chiral arrangement, which can occur through intrachain and interchain interaction in the aggregate state [24]. The CD spectrum for the reduced polymer reveals a split-type Cotton effect, while that in the oxidized state does not. The observation of such a band for the polymer may suggest the presence of an intermolecular process upon aggregate formation [15]. In this case, the bisignate (two-splited) couplet in the CD spectra indicates a onehanded helical packing of the polymer chains, with a positive couplet for poly1 and a negative couplet for poly2. This electrochemical doping-dedoping induced change in $\mathrm{CD}$ for the polymer can therefore be reasonably explained as being due to the intercalation of the perchlorate ion and solvent between individual main chains at the molecular level, resulting in extension of the distance between the main chains in the oxidized state. As shown in Fig. 4, poly2 in 0.1M TBAP/acetonitrile solution underwent a reversible change in ellipticity at $560 \mathrm{~nm}$ with application of triangular wave produced by cyclic voltammetry. Thus, sequential change in applied voltage for the 


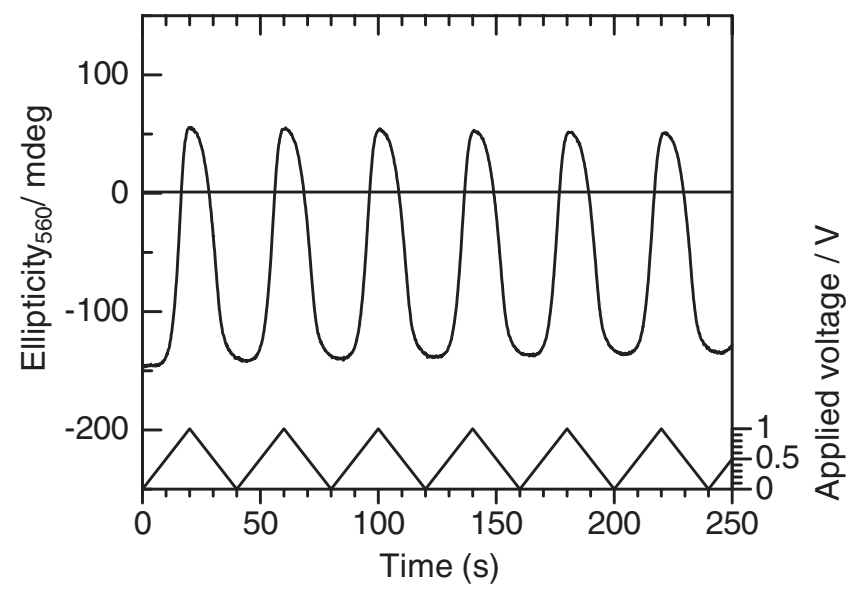

FIG. 4. Reversible change in ellipticity at $560 \mathrm{~nm}$ for an electrochromic cell based on poly 2 as a function of applied voltages between 0 and $1 \mathrm{~V}$.

polymers allows reversible change in $\mathrm{CD}$ intensity through the doping-dedoping process.

Poly1 and poly2 exhibit maximum optical rotations of $-1.7 \times 10^{4}$ and $+1.9 \times 10^{4} \mathrm{deg} / \mathrm{cm}$ at $473 \mathrm{~nm}$ $(-0.07 \mathrm{~V})$, respectively. This property is comparable to the functionality of a Kerr device and Faraday optical rotator. The modulation of optical rotation observed in this polymer originates from the helical macromolecular arrangement produced by imprinting of the structure chirality of the CLC electrolyte [25]. The control of the optical activity of the polymer is thus driven by the electrochemical doping-dedoping process.

In conclusion, the preparation of the polymers with structure chirality was shown experimentally to provide intense CD and optical rotation. The natural optical activity of the polymers was successfully controlled by the electrochemical doping-dedoping procedure. The finding of this unprecedented mechanism, involving doping of $\pi$-conjugated systems, can be an important advance in the development of plastic optoelectronics based on conductive polymers.

The author would like to thank Professor Kazuo Akagi (Kyoto University) for synthesis of No. PCH-506 binol.

*gotoh@ims.tsukuba.ac.jp

[1] W. Lu, A. G. Fadeev, B. Qi, E. Smela, B. R. Mattes, J. Ding, G. M. Spinks, J. Mazurkiewicz, D. Zhou, G. G. Wallace, D. R. MacFarlane, S. A. Forsyth, and M. Forsyth, Science 297, 983 (2002).

[2] H. Shirakawa, E. Louis, A. G. MacDiarmid, C. K. Chiang, and A. J. Heeger, J. Chem. Soc. Chem. Commun. doi:10.1039/c39770000578 (1977).

[3] S. A. Sapp, G. A. Sotzing, J.L. Reddinger, and J.R. Reynolds, Adv. Mater. 8, 808 (1996).
[4] A. A. Argun, P.-H. Aubert, B. C. Thompson, I. Schwendeman, C. L. Gaupp, J. Hwang, N. J. Pinto, D. B. Tanner, A. G. MacDiarmid, and J. R. Reynolds, Chem. Mater. 16, 4401 (2004).

[5] A. A. Argun, A. Cirpan, and J. R. Reynolds, Adv. Mater. 15, 1338 (2003).

[6] S. Courty, A. R. Tajbakhsh, and E. M. Terentjev, Phys. Rev. Lett. 91, 085503 (2003).

[7] S. Courty, A. R. Tajbakhsh, and E. M. Terentjev, Phys. Rev. E 73, 011803 (2006).

[8] M. Mitov and N. Dessaud, Nat. Mater. 5, 361 (2006).

[9] I. Tinoco and M. P. Freeman, J. Phys. Chem. 61, 1196 (1957).

[10] K. Akagi, G. Piao, K. Sakamaki, H. Shirakawa, and M. Kyotani, Science 282, 1683 (1998).

[11] K. Akagi, S. Guo, T. Mori, M. Goh, G. Piao, and M. Kyotani, J. Am. Chem. Soc. 127, 14647 (2005).

[12] H. Goto and K. Akagi, Macromolecules 38, 1091 (2005).

[13] The film thickness of the polymer was measured with a ZYGO newview5032 3-D surface structure analysis microscope with frequency domain analysis method.

[14] G. Sonmez, C. K. F. Shen, Y. Rubin, and F. Wudl, Angew. Chem., Int. Ed. 43, 1498 (2004).

[15] B. M. W. Langeveld-Voss, R. A. J. Janssen, and E.W. Meijer, J. Mol. Struct. 521, 285 (2000).

[16] J. J. L. M. Cornelissen, A.E. Rowan, R. J. M. Nolte, and N. A. J. M. Sommerdijk, Chem. Rev. 101, 4039 (2001).

[17] T. Miteva, L. Palmer, L. Kloppenburg, D. Neher, and U. H.F. Bunz, Macromolecules 33, 652 (2000).

[18] R. Fiesel and U. Scherf, Macromol. Rapid Commun. 19, 427 (1998).

[19] S. J. George, A. Ajayaghosh, P. Jonkheijm, A. P. H. J. Schenning, and E. W. Meijer, Angew. Chem., Int. Ed. 43, 3422 (2004).

[20] R. Fiesel, C. E. Halkyard, M. E. Rampey, L. Kloppenburg, S. L. Studer-Martinez, U. Scherf, and U.H.F. Bunz, Macromol. Rapid Commun. 20, 107 (1999).

[21] D. Iarossi, A. Mucci, F. Parenti, L. Schenetti, R. Seeber, C. Zanardi, A. Forni, and M. Tonelli, Chem. Eur. J. 7, 676 (2001).

[22] C. E. Halkyard, M.E. Rampey, L. Kloppenburg, S. L. Studer-Martinez, and U.H.F. Bunz, Macromolecules 31, 8655 (1998).

[23] O. Henze, W. J. Feast, F. Gardebien, P. Jonkheijm, R. Lazzaroni, Ph. Leclere, E. W. Meijer, and A. P. H. J. Schenning, J. Am. Chem. Soc. 128, 5923 (2006).

[24] F. D. Lewis, L. Zhang, X. Liu, X. Zuo, D. M. Tiede, H. Long, and G. C. Schatz, J. Am. Chem. Soc. 127, 14445 (2005).

[25] Cholesterics generally shows extraordinary large optical rotation (ca. $10^{3}-10^{4} \% \mathrm{~cm}$ ). For example, chiral crystals, such as berg crystal displays optical rotation of $250^{\circ} / \mathrm{cm}$. Optically active molecules show optical rotation of several dozen degrees $/ \mathrm{cm}$. This large optical rotation of cholesterics derives not from optical rotatory power of the individual chiral molecules but from chiral architectures. The individual molecules of the polymer, prepared in the CLC electrolyte, are also arranged in CLC-like macroscopic helical formation. 